\title{
POLÍtICAS DE SAÚdE E EdUCAÇÃO PARA A JUVENTUdE NO BRASIL: INTERSETORIALIDADE E ATUAÇÃO DO ENFERMEIRO
}

HEALTH AND EDUCATION POLICIES FOR YOUTH IN BRAZIL: INTERSECTORIALITY AND NURSES ROLE

POLÍTICAS DE SALUD Y EDUCACIÓN PARA LA JUVENTUD EN BRASIL: INTERSECTORIALIDAD Y DESEMPEÑO DEL ENFERMERO

Emanoel Avelar Muniz ${ }^{1}$

Maria Veraci Oliveira Queiroz ${ }^{2}$

Francisco Clécio da Silva Dutra ${ }^{3}$

Aretha Feitosa de Araújo 4

Lucilane Maria Sales da Silva ${ }^{5}$

Raimundo Augusto Martins Torres 6

Como Citar:

Muniz EA, Queiroz MVO, Dutra FCS, Araújo AF, Silva LMS, Torres RAM. Políticas de saúde e educação para a juventude no brasil: intersetorialidade e atuação do enfermeiro. Sanare (Sobral, Online). 2021;20(1):73-80.

Palavras-chave:

Política Pública; Saúde do Adolescente; Adulto Jovem; Promoção da Saúde; Enfermagem.

Keywords:

Public Policy; Adolescent Health;

Young Adult; Health Promotion;

Nursing.

Palabras clave:

Política Pública; Salud del joven;

Adulto y joven; Promoción de la salud; Enfermería.

Submetido: $24 / 08 / 2020$

Aprovado: 14/06/2021

Autor(a) para Correspondência: Emanoel Avelar Muniz E-mail:emanoel.muniz@ifce.edu.br

Cert. de Redação Científica: Central das Revisões. Edição de texto: Karina Maria M. Machado. Revisão de provas: Texto definitivo validado pelos (as) autores(as).

\section{RESUMO}

Experiências internacionais mostram que os enfermeiros realizam intervenções na escola para promover a saúde e o sucesso acadêmico dos estudantes. Este estudo objetiva refletir sobre a intersetorialidade das políticas de saúde e educação para a juventude no Brasil e a atuação do enfermeiro na saúde escolar com jovens. Trata-se de um ensaio teórico fundamentado em políticas brasileiras de saúde e educação para jovens, e em estudos científicos. No Brasil, políticas que integram saúde e educação para a juventude foram construídas, essencialmente, ao final da década de 1980, pautadas na atenção integral aos jovens. Mais recentemente, a criação do Programa Saúde na Escola e do Programa Nacional de Assistência Estudantil vem propiciando aos profissionais de enfermagem assumir mais diretamente ações de proteção e promoção à saúde dos jovens em ambientes educacionais. Apesar de alguns programas incluírem diretrizes que articulam as políticas de saúde e educação no Brasil, há fragilidade nas ações intersetoriais e, notadamente, na atuação do enfermeiro com jovens em ambientes educacionais, o que demonstra a necessidade de ampliação das práticas de enfermagem escolar.

1. Enfermeiro, doutorando do Programa de Pós-graduação Cuidados Clínicos em Enfermagem e Saúde (PPCCLIS) da Universidade Estadual do Ceará (UECE).E-mail: emanoel.muniz@ifce.edu.br 0RCID: https://orcid.org/0000-0002$\underline{8648-8523}$

2. Enfermeira, Doutora em Enfermagem pela Universidade Federal do Ceará (UFC), docente do PPCCLIS e da graduaçãoem enfermagem da UECE. E-mail: maria.queiroz@uece.br ORCID: https://orcid.org/0000-0002-7757$\underline{119 x}$

3. Enfermeiro, doutorando do PPCCLIS da UECE. E-mail: cleciouece@gmail.com ORCID: https://orcid.org/0000$\underline{0002-3451-1664}$

4. Enfermeira, doutoranda do PPCCLIS da UECE. E-mail: aretha.feitosa@gmail.com 0RCID: https://orcid.orq/00000001-9297-8281

5. Enfermeira, Doutora em Enfermagem pela Universidade Federal do Ceará (UFC), docente do PPCCLIS e da graduação em enfermagem da UECE. E-mail: lucilanemaria@yahoo.com.br ORCID: https://orcid.org/0000-0002$\underline{3850-8753}$

6. Enfermeiro, Doutor em Educação pela UFC, docente do PPCCLIS e da graduação em enfermagem da UECE. E-mail: augustomtorres70@gmail.com 0RCID: https://orcid.org/0000-0002-8114-4190 


\section{ABSTRACT}

International experiences show that nurses perform interventions in schools to promote health and academic success of the students. This study aims to reflect on the intersectoriality of health and education policies for youth in Brazil and the role of nurses in school health with young people. This is a theoretical essay based on Brazilian health and education policies for young people and on scientific studies. In Brazil, policies that integrate health and education for the youth were built, essentially, at the end of the 1980s, based on comprehensive care for young people. More recently, the creation of the Health at School Program and the National Student Assistance Program has enabled nursing professionals to take more direct action to protect and promote health of young people in educational environments. Although some programs include guidelines that articulate health and education policies in Brazil, there is a weakness in intersectoral actions and, notably, in the role of nurses with young people in educational environments, therefore, it is necessary to expand school nursing practices.

\section{RESUMEN}

Las experiencias internacionales muestran que los enfermeros realizan intervenciones en la escuela que promuevan la salud y el éxito académico de los estudiantes. Este estudio tiene como objetivo reflexionar sobre la intersectorialidad de las políticas públicas sanitarias y educación para la juventud en Brasil y la actuación del enfermero en la salud de los escolares. Se trata de un ensayo teórico basado en políticas brasileñas de salud y educación para jóvenes y en estudios científicos. En Brasil, políticas que integran salud y educación para la juventud fueron construidas, esencialmente, al final de la década de 1980, pautadas en la atención integral a los jóvenes. Más recientemente, la creación del Programa Salud en la Escuela y del Programa Nacional de Asistencia Estudiantil viene proporcionando a los profesionales de enfermería que asuman más directamente acciones de protección y promoción a la salud de los jóvenes en espacios educacionales. A pesar de que algunos programas incluyan directrices que articulan las políticas de salud y educación en Brasil, hay debilidad en las acciones intersectoriales $y$, notablemente, en la actuación del enfermero con jóvenes en ambientes educacionales, por consiguiente, es necesario ampliar las prácticas de enfermería escolar.

\section{INTRODUÇÃO}

A Organização Mundial da Saúde (OMS) utiliza o termo "pessoas jovens" para os sujeitos que estão na faixa etária de 10 a 24 anos de idade, incluindo adolescentes e jovens ${ }^{1}$. No Brasil, o Estatuto da Juventude define jovens em três grupos: 15 a 17 anos de idade, denominados jovens-adolescentes; 18 a 24 anos de idade, como jovens-jovens; e 25 a 29 anos de idade, como jovens adultos ${ }^{2}$. Informações oficiais mostram que, em 2014, existiam 48,9 milhões de jovens de 15 a 29 anos no Brasil, correspondendo a $23,6 \%$ da população. Por outro lado, observa-se crescente morbimortalidade por causas externas nessa faixa etária, com destaque para as agressões, acidentes com transportes terrestres e suicídios. Há exposição elevada de jovens a fatores de risco como álcool, violência, sexo desprotegido e predomínio de hábitos não saudáveis ${ }^{3,4}$.

Além disso, o baixo nível educacional continua sendo o principal fator de risco para o futuro dos jovens e parece que as políticas de saúde e educação de caráter universal têm sido insuficientes em sua gestão para promover equidade de acesso e permanência na escola, com repercussões nas condições de vida e saúde ${ }^{5}$. Assim, propõese o desenvolvimento da intersetorialidade das políticas estruturais, programas e ações para a promoção do bem-estar e desenvolvimento integral da juventude. A intersetorialidade é definida como um princípio da Política Nacional de Promoção da Saúde (PNPS) e se refere ao processo de articulação de saberes, potencialidades e experiências de sujeitos, grupos e setores na construção de intervenções compartilhadas, estabelecendo vínculos, corresponsabilidade e cogestão para objetivos comuns ${ }^{6}$.

Nas últimas décadas, movimentos de superação da setorialidade nos campos da saúde e da educação incluem ações de educação e promoção da saúde aos estudantes. A exemplo, o Ministério da Saúde (MS) implementou programas, estratégias 
e políticas para diversos grupos etários, pois o ambiente escolar assume espaço privilegiado para a execução dessas ações?

No Brasil, as políticas criadas mais recentemente, que articulam os setores saúde e educação, foram o Programa Saúde na Escola (PSE), instituído em 2007, que objetiva contribuir para a formação integral dos estudantes da rede pública de educação básica por meio de ações de prevenção de doenças e agravos, promoção e atenção à saúde, e o Programa Nacional de Assistência Estudantil (PNAES), instituído em 2010, este com a finalidade de ampliar as condições de permanência dos jovens na educação profissional e superior pública federal ${ }^{8,9}$. 0 s referidos programas incluem o trabalho do enfermeiro em dimensões colaborativas com outros profissionais. Nesse contexto, considera-se que a enfermagem, quando inserida no ambiente escolar, desenvolve ações estratégicas que podem promover a saúde dos jovens. Entretanto, para demonstrar sua relevância, é necessário que a enfermagem escolar evolua no atendimento das necessidades atuais de educação e de saúde ${ }^{10}$.

Experiências internacionais mostram que os enfermeiros realizam intervenções com alunos, pais e funcionários da escola para promover a saúde e o sucesso acadêmico dos estudantes. Essas atividades contribuem para a diminuição do absenteísmo, aumento da adesão à imunização, melhoria do manejo de condições crônicas, prevenção de doenças, apoio na identificação e gerenciamento de problemas mentais e questões de saúde, bem como suporte psicossocial, permitindo assim que os estudantes permaneçam na escola e melhorem seu desempenho acadêmico ${ }^{11,12}$.

Em escolas onde há enfermeiro ou serviço de enfermagem, observa-se um impacto na articulação interdisciplinar. 0 enfermeiro escolar é o profissional treinado para prestar cuidados de enfermagem especializados de forma autônoma, no campo educacional, em uma equipe interdisciplinar. Portanto, um papel intersetorial está entre os novos atributos de identidade de ser enfermeiro. Assim, a atuação desse profissional nas instituições educacionais torna-se uma necessidade social e de saúde, de modo que a presença física dos enfermeiros representa o cuidado aos estudantes ${ }^{13}$.

No Brasil, estudo mostra que mesmo existindo diretrizes para o trabalho da enfermagem na escola, a indisponibilidade de profissionais para o desenvolvimento da enfermagem escolar impossibilita efetivar ações integradas, sendo este um impeditivo para o planejamento e continuidade da gestão intersetorial ${ }^{14}$.

Considerando o exposto, este estudo teve como objetivo refletir sobre a intersetorialidade das políticas de saúde e educação para a juventude no Brasil e a atuação do enfermeiro na saúde escolar com os jovens. Realizou-se um resgate das principais políticas que articulam saúde e educação para os jovens desenvolvidas recentemente no Brasil, destacando suas interfaces, e pesquisas que discorrem sobre a atuação do enfermeiro, no contexto escolar, em âmbitos nacional e internacional. Espera-se que as reflexões ampliem o olhar sobre a importância dessa integração e potencializem ações intersetoriais para a melhoria da atenção e cuidado aos jovens, sendo, portanto, a escola um campo de atuação da enfermagem no exercício e colaboração interprofissional.

\section{POLÍTICAS DE SAÚdE E EDUCAÇÃO PARA A JUVENTUDE NO BRASIL}

No Brasil, inegavelmente, nos últimos anos, houve avanços consideráveis em relação à construção de um rol significativo de regulamentações, políticas, programas e instituições, ou seja, de ações voltadas à promoção da juventude, defesa dos interesses e direitos do público jovem. Pesquisadores da educação concordam que, a partir da segunda metade do século XX, ocorreu rápida expansão do sistema educacional brasileiro, registrando-se a ampliação de oportunidades de acesso e escolarização da população brasileira, essencialmente, para os segmentos mais novos da sociedade nas etapas da educação básica 5 .

A ampliação e garantia desses direitos foram asseguradas no Estatuto da Criança e do Adolescente (ECA) de 1990 e na Lei 8.080, também de 1990, que rege o Sistema Único de Saúde (SUS) ${ }^{15}$. Várias iniciativas com esse propósito foram se concretizando no panorama nacional em cumprimento à Constituição Federal de 1988 para assegurar direitos fundamentais em benefício ao desenvolvimento dos adolescentes e jovens. Nomeadamente, para essa população, o Ministério da Saúde oficializou, em 1989, o Programa Saúde do Adolescente (PROSAD), que teve a finalidade de promover, integrar, apoiar e incentivar práticas nos locais onde eram realizadas atividades para esse público, sejam nos estados, municípios, 
universidades, organizações não-governamentais e outras instituições, incluindo a escola ${ }^{16}$.

No entanto, estudo indica que esse Programa não tem atendido a todos os adolescentes brasileiros, há fragilidade na articulação de ações para contemplar o público-alvo, pouca variabilidade de metas e desafios na participação ativa dos adolescentes. Destacam-se, entretanto, como potencialidades do Programa, a capacitação profissional promovida para o atendimento ao adolescente e o reconhecimento deste como protagonista nas ações em saúde ${ }^{17}$.

Compreendendo que muitas dessas ações concretizam-se nos serviços de saúde e na articulação com a escola, em continuidade e reforçando as práticas de proteção à saúde dessa população, destaca-se o Projeto Saúde e Prevenção nas Escolas (PSPE), instituído pelos Ministérios da Educação (MEC) e da Saúde, contando com o apoio da Organização das Nações Unidas (ONU), desde o ano de 2003. Representa, portanto, um marco na integração saúde-educação e destaca a escola como - espaço ideal para a articulação das políticas voltadas para adolescentes e jovens ${ }^{18}$.

0 PSPE desponta como uma potencial estratégia de articulação intersetorial e interprofissional, interagindo com a sociedade civil organizada e a comunidade escolar, cujas ações podem ser desenvolvidas nos territórios definidos segundo a área de abrangência da Estratégia Saúde da Família (ESF), possibilitando um movimento de colaboração entre os campos da saúde e da educação e seus equipamentos ${ }^{7}$.

Em 2007, o PSE foi instituído por meio do Decreto $n .^{0} 6.286$ e constitui estratégia para a integração e a articulação permanente entre as políticas e ações de educação e de saúde, com a participação da comunidade escolar, envolvendo as equipes de Saúde da Família e da educação básica ${ }^{8}$. Ações integradas como as propostas pelo PSE contribuem para a formação integral e podem ampliar o alcance e efeito nas condições de vida e saúde dos estudantes, famílias e comunidade ${ }^{19}$.

Para fortalecer a atenção e o cuidado a esse público, foram lançadas, em 2010, as Diretrizes Nacionais para a Atenção Integral à Saúde de Adolescentes e Jovens, na promoção, proteção e recuperação da saúde, com o objetivo de sensibilizar e mobilizar gestores e profissionais do SUS para integrar ações, programas e políticas por meio de estratégias interfederativas e intersetoriais.
As diretrizes reforçam o fortalecimento da promoção da saúde e a reorientação dos serviços de saúde, e trazem como eixos estruturantes das ações: a) acompanhamento do crescimento e desenvolvimento; b) atenção integral à saúde sexual e à saúde reprodutiva; e c) atenção integral ao uso abusivo de álcool e outras drogas por pessoas jovens ${ }^{15}$.

Além disso, considerando a baixa frequência de jovens no ensino superior, foram criadas ações afirmativas para corrigir, parcialmente, as desvantagens quanto ao ingresso e permanência de alguns grupos sociais. Dentre elas, destacase o Programa Nacional de Assistência Estudantil (PNAES), instituído pelo Decreto n. ${ }^{0} 7.234$ de $2010^{\circ}$. A assistência estudantil é composta por uma variedade de eixos de atuação, incluindo a atenção integral à saúde. Assim, a proposta do PNAES é articular diferentes áreas e, consequentemente, diferentes políticas sociais.

No entanto, destacam-se no PNAES algumas limitações, dentre elas: a ausência de ações conjuntas entre o Ministério da Educação e Ministério da Saúde; falta de capacitação dos profissionais da assistência estudantil em temas de saúde; não inclusão dos estudantes de pós-graduação; ausência de diretrizes sobre a organização das ações de saúde, bem como de mecanismos de monitoramento e avaliação, além de desarticulação com a rede de saúde onde está inserida a instituição de ensino ${ }^{20}$.

Diante do recorte apresentado sobre as principais políticas de saúde e educação para a juventude no Brasil, reconhece-se que essas priorizam os jovens em situação de vulnerabilidade social, embora alguns programas e ações contemplem essa população de uma maneira geral. Além disso, não bastam a aproximação entre os setores saúde e educação e a formulação de políticas conjuntas para a efetivação de ações intersetoriais, é preciso avançar na reorientação das práticas de formação e participação social no SUS para que as ações sejam, de fato, integradas ${ }^{14}$.

Assim, considerando que saúde e educação são áreas reconhecidas como produtoras de qualidade de vida e transformação social, destacase a necessidade de manter e implementar ações intersetoriais pautadas nessas e em outras políticas que atendam às demandas e necessidades dos jovens, propiciando oportunidades de desenvolvimento pessoal e profissional, com repercussões na 
vida e na saúde. Destaca-se a diminuição de investimentos em saúde e educação nos últimos anos, no Brasil, afetando o financiamento das ações e, consequentemente, a atuação do enfermeiro na escola. Assim, os contextos sociais e políticos atuais desfavorecem ou impedem que esses programas sejam amplamente efetivados.

\section{BREVE PANORAMA DA ATUAÇÃO DO ENFERMEIRO NA SAÚDE ESCOLAR COM JOVENS}

0 papel do enfermeiro escolar varia entre os países, porém o objetivo de sua atuação é identificar necessidades, melhorar a saúde e o bem-estar dos alunos. Estudo norte-americano mostra que enfermeiros escolares prestam cuidados de saúde qualificados para estudantes, realizam triagens, acompanhamentos e encaminhamentos para outros serviços e profissionais de acordo com a necessidade, promovendo o acesso à saúde principalmente na população em situação de vulnerabilidade ${ }^{11}$.

Nos Estados Unidos, a maioria das intervenções de enfermagem realizadas no ambiente escolar estão relacionadas ao princípio da coordenação do cuidado para atender aos objetivos, necessidades e expectativas dos estudantes e família, com destaque para entrevistas motivacionais, aconselhamento e comunicação colaborativa com pais, funcionários da escola, outros profissionais de saúde e comunidade. As intervenções também foram relacionadas a melhores resultados de saúde e educação dos alunos ${ }^{12}$.

Estudo realizado no Peru destaca que dentro das competências do enfermeiro escolar está a articulação da educação com a saúde, em que se reúnem a aprendizagem acadêmica escolar e a aprendizagem para a vida, saúde e bem-estar, no contexto de programas de saúde escolar, realizados por profissionais da saúde advindos de serviços de atenção primária ou de serviços exclusivos de saúde escolar, enfatizando a promoção da saúde ${ }^{13}$.

No Brasil, as ações de saúde desenvolvidas com - público jovem nas escolas alteram a dinâmica escolar e se aproximam do conceito de promoção da saúde, mas, às vezes, o profissional não está preparado para a interação com os jovens quando não há integração dos serviços. Assim, a participação da comunidade educativa, incluindo profissionais da educação, estudantes e familiares, é importante em todas as etapas das ações de saúde nas escolas ${ }^{21}$. Boas perspectivas foram demonstradas para o exercício da enfermagem brasileira na área da saúde escolar, entretanto evidenciou-se a necessidade de fortalecer essa área por meio de uma integração efetiva entre saúde e educação, visando à prestação de uma assistência integral ao escolar 22 .

Salienta-se que a presença do enfermeiro na escola já acontece há algumas décadas, independentemente da criação de políticas intersetoriais, e a literatura ressalta que seu trabalho é determinante para desencadear ações de promoção da saúde, gerar discussões, estimular debates, realizar orientações e apresentar perspectivas em relação ao processo saúde-doença da comunidade, além de fortificar as relações sociais entre os profissionais da educação e da saúde ${ }^{23}$. A enfermagem se destaca como categoria do setor saúde responsável pela qualidade da assistência, com uso das tecnologias da informação e comunicação no cenário da saúde coletiva, incluindo as escolas e demais instituições educacionais a serviço das juventudes ${ }^{24}$.

No âmbito do PSE, os enfermeiros realizam ações educativas em saúde com grande potencial de responder às necessidades de saúde escolar dos jovens, uma vez que o programa tem propósitos de promover boas condições de saúde para 0 desenvolvimento integral dos estudantes. Portanto, é preciso avançar em inovações tecnológicas, no âmbito das práticas do PSE, que ressignifiquem a escola como cenário da promoção da saúde ${ }^{25}$.

Ademais, no desenvolvimento de atividades que promovam a saúde no espaço escolar, devese considerar que este é permeado por relações sociais constantes, elucidando a necessidade de colaboração interprofissional'. Nesse sentido, observam-se lacunas de cuidados destinados aos jovens e dificuldades na implementação do PSE, como o desconhecimento dos profissionais sobre o programa, a celeridade na sua implantação, com frágil formação dos profissionais, além das condições estruturais das escolas, recursos humanos e materiais e a falta de planejamento das ações, confirmando a desarticulação dos setores educação e saúde. A integração da tríade equipe de saúde, escola e família configura potencialidade do programa 26,13 .

Diante do exposto, percebe-se que no Brasil o enfermeiro tem uma discreta atuação em 
programas que integram saúde e educação, mas vem aproximando-se desse cuidado, embora de forma pontual e com uma evolução gradativa. Acredita-se que as lacunas existentes têm como desafios superar ações programáticas pontuais e avançar para um agir integrado pela colaboração interprofissional e intersetorial que atenda às necessidades dos jovens.

Acrescenta-se que existem lacunas de evidências sobre a eficácia das práticas de enfermagem escolar, incluindo o impacto no desempenho acadêmico dos estudantes, gerando um debate sobre o papel e a necessidade de enfermeiros escolares no sistema de ensino atual ${ }^{10}$. É necessário que os estudos evoluam para além das descrições das intervenções, mostrando evidências sobre os seus efeitos.

No território brasileiro, apesar de existirem ações de saúde na escola, na perspectiva intersetorial, ocorrem impasses na sua regulação, dificultando a implementação, pois não se percebe a inclusão necessária do enfermeiro nesse espaço, mesmo reconhecendo-se o seu potencial na promoção da saúde de jovens. Acrescenta-se que, para 0 fortalecimento dessas ações, é imprescindivel o apoio da gestão para que haja planejamento e ações contínuas e sistemáticas, com padronização das práticas devido à diversidade de propostas existentes. Além disso, é preciso propiciar formação acadêmica e educação permanente que contemplem habilidades e competências dos enfermeiros em ações multiprofissionais com jovens no ambiente escolar.

\section{CONCLUSÃO}

Propostas de desenvolvimento de programas com ações de saúde na escola existem há décadas no Brasil; contudo, as políticas públicas brasileiras envolvendo a intersetorialidade saúde e educação, com destaque à proteção e desenvolvimento de adolescentes e jovens, vêm definindo-se a partir da constituição de 1988 e são incentivadas também pelos organismos internacionais ante à necessidade de promoção à saúde da juventude em ambientes educacionais. Nesse aspecto, identificaram-se programas com propósitos e ações intersetoriais envolvendo saúde e educação, destacadamente, - PSE e o PNAES, que sinalizam possibilidades de trabalhar temas de interesses comuns direcionados aos estudantes, sobretudo àqueles em situações de vulnerabilidades, que podem impactar nas suas condições de saúde e aprendizado.

É compreensível que ações intersetoriais serão efetivadas mediante a colaboração interprofissional envolvendo, essencialmente, as equipes de Saúde da Família e/ou de assistência estudantil, integradas com educadores responsáveis pela formação integral dos jovens. Mas também é preciso ouvir os jovens, planejar e desenvolver ações com os mesmos, incentivando o protagonismo juvenil. Assim, a inserção do enfermeiro no ambiente escolar precisa ganhar maior visibilidade nos espaços acadêmicos e profissionais, bem como o apoio dos gestores com decisões políticas. Espera-se que as reflexões contribuam para um novo olhar sobre essa atuação e a necessidade de fortalecer e definir as práticas de enfermagem em ambientes educacionais.

Destaca-se como limitação a reduzida abrangência dos estudos, ou seja, um delineamento que proporcione, além da reflexão, identificar as intervenções de enfermagem realizadas com jovens escolares e os seus efeitos, ampliando-se o debate sobre a prática do enfermeiro escolar e o impacto nos resultados de saúde e educação dos estudantes.

\section{CONTRIBUIÇÃO DOS AUTORES}

Emanoel Avelar Muniz e Maria Veraci Oliveira Queiroz contribuíram na concepção do estudo, construção do ensaio e revisão final do manuscrito. Francisco Clécio da Silva Dutra e Aretha Feitosa de Araújo contribuíram na revisão final do manuscrito. Raimundo Augusto Martins Torres contribuiu na concepção do estudo e revisão final do manuscrito. Lucilane Maria Sales da Silva contribuiu na concepção do estudo.

\section{REFERÊNCIAS}

1. World Health Organization. Young People's Health - a Challenge for Society. Report of a WHO Study Group on Young People and Health for All. Technical Report Series 731. Geneva: WHO; 1986.

2. Brasil. Estatuto da juventude: atos internacionais e normas correlatas. Brasília (DF): Senado Federal; 2013.

3. Instituto Brasileiro de Geografia e Estatística. Síntese de indicadores sociais: uma análise das condições de vida da população brasileira 2016. Rio de Janeiro: IBGE; 2016.

4. Reis AAC, Malta DC, Furtado LAC. Challenges for public policies aimed at adolescence and 
youth based on the National Scholar Health Survey (PeNSE). Ciênc Saúde Colet [serial on the internet]. 2018 Sep [cited 2019 Dec 19];23(9):2879-90. Available from: http://www.scielo.br/scielo. php?script $=$ sci arttext\&pid $=\$ 14138123201800090$ 2879\&lng $=$ en

5. Sposito MP, Souza R, Silva, FA. The research on young people in Brazil: setting new challenges from quantitative data. Educ Pesqui [serial on the internet]. 2018 [cited 2019 Nov 06];44: e170308. Available from http://www.scielo.br/scielo.php?script=sci arttext\&pid=S1517970220180001004 $\underline{30 \& \operatorname{lng}=e n \& n r m=i s 0}$

6. Brasil. Política Nacional de Promoção da Saúde: PNPS: Anexo I da Portaria de Consolidação $n^{0}$ 2 , de 28 de setembro de 2017, que consolida as normas sobre as políticas nacionais de saúde do SUS [document on the internet]. Brasilia (DF): Ministério da Saúde; 2018 [cited 2020 June 25]. Available from: https://bvsms.saude.gov.br/bvs/ publicacoes/politica nacional promocao saude. $\underline{p d f}$

7. Dias MSA, Vieira FMBR, Silva LCC, Vasconcelos MI0, Machado MFAS. Colaboração interprofissional no Projeto Saúde e Prevenção na Escola. Ciênc Saúde Colet [serial on the internet]. 2016 June [cited 2020 June 25];21(6):1789-98. Available from: http://www.scielo.br/scielo . php?script =sci arttext\&pid =S1413$81232016000601789 \& \operatorname{lng}=$ en

8. Brasil. Decreto $n^{\circ} 6.286$, de 5 dez. 2007. Institui - Programa Saúde na Escola - PSE, e dá outras providências. Diário Oficial da União [document on the internet]. 5 de dezembro de 2007 [cited 2020 June 25]; Seção 1. Available from: http://www. planalto.gov.br/ccivil 03/ ato2007-2010/2007/ decreto/d6286.htm

9. Brasil. Decreto $n^{0}$ 7.234, de 19 de jul. de 2010. Dispõe sobre o Programa Nacional de Assistência Estudantil - PNAES. Diário Oficial da União [document on the internet]. 20 de julho de 2010 [cited 2020 June 25]; Seção 1. Available from: https://www2.camara.leg.br/legin/fed/ decret/2010/decreto-7234-19-julho-2010-607316norma-pe.html

10. Doi L, Wason D, Malden S, Jepson R. Supporting the health and well-being of school-aged children through a school nurse programme: a realist evaluation. BMC Health Serv Res [serial on the internet]. 2018 [cited 2019 Nov 06];18(664):110. Available from: https://bmchealthservres. biomedcentral.com/track/pdf/10.1186/s12913$\underline{018-3480-4}$

11. Maughan ED, Cowell J, Engelke MK, McCarthy $A M$, Bergren MD, Murphy MK, et al. The vital role of school nurses in ensuring the health of our nation's youth. Nurs Outlook [serial on the internet]. 2018 [cited 2019 Nov 06];66(1):94-6. Available from: https://www.sciencedirect.com/ $\mathrm{s}$ c i e n c e / a r t i c l e / p i i / S0029655417306267?via\%3Dihub

12. Best NC, Oppewal S, Travers D. Exploring School Nurse Interventions and Health and Education Outcomes: An Integrative Review. J Sch Nurs. 2018;34(1):14-27. doi: https://doi. org/10.1177/1059840517745359

13. Mori FMLV, Edquén SB, Espinoza ZEL, Salazar RS. Competencias de la enfermera en instituciones educativas: una mirada desde los gestores educativos. Rev Gaúcha Enferm. 2018; 39:e20170152. doi: https://doi.org/10.1590/1983$\underline{1447.2018 .2017-0152}$

14. Brasil EGM, Silva RM, Silva MRF, Rodrigues DP, Queiroz MVO. Adolescent health promotion and the School Health Program: complexity in the articulation of health and education. Rev esc enferm USP [serial on the internet]. 2017 [cited 2019 Nov 06];51:e03276. Available from: https:// www.scielo.br/pdf/reeusp/v51/1980-220X-reeuspS1980-220X2016039303276.pdf

15. Brasil. Diretrizes Nacionais para a Atenção Integral à Saúde de Adolescentes e Jovens na Promoção, Proteção e Recuperação da Saúde. Brasília (DF): Ministério da Saúde; 2010.

16. Brasil. Programa Saúde do Adolescente: Bases Programáticas. $2^{a}$ ed. Brasília: Ministério da Saúde; 1996.

17. Jager ME, Batista FA, Perrone CM, SAntos SS, Disas ACG. 0 adolescente no contexto da saúde pública brasileira: reflexões sobre o PROSAD. Psicol estud [serial on the internet]. 2014 [cited 2019 Nov 05];19(2):211-21. doi: http://dx.doi. org/10.1590/1413-737221567004

18. Simões CA, Moll J, Malheiro MFS, Oliveira MAK. Programas de promoção de saúde integrados na política nacional de educação: o papel da escola na prevenção do uso de drogas. In: Sudbrack MF0, Conceição MIG, Costa LF. Curso de prevenção do uso de drogas para educadores de escolas públicas. 6. ed. Brasília: Ministério da Justiça, Ministério da Educação; 2014.

19. Lopes IE, Nogueira JAD, Rocha DG. Axes of action of the School Health Program and Health Promotion: an integrative review. Saúde debate [serial on the internet]. 2018 [cited 2019 Nov 05]; 42(118):77389. Available from: https://www.scielo.br/pdf/ sdeb/v42n118/0103-1104-sdeb-42-118-0773.pdf 
20. Bleicher T, Oliveira RCN. Políticas de assistência estudantil em saúde nos institutos e universidades federais. Psicologia Escolar e Educacional. 2016;20(3):543-49. Available from: https://dx.doi. org/10.1590/2175-3539201502031040

21. Carvalho FFB. A saúde vai à escola: a promoção da saúde em práticas pedagógicas. Physis [serial on the internet]. 2015 Dec [cited 2019 Nov 01];25(4):1207-27. Available from: https:// www.scielo.br/pdf/physis/v25n4/0103-7331physis-25-04-01207.pdf

22. Pires LM, Queirós PS, Munari DB, Melo CF, Souza MM. A enfermagem no contexto da saúde do escolar: revisão integrativa da literatura. Rev enferm UERJ [serial on the internet];2012 Dez [cited 2019 Nov 01];20(1):668-75. Available from: https://www.epublicacoes.uerj.br/index.php Lenfermagemuerj/article/view/5968/4284

23. Rasche AS, Santos MSS. Enfermagem escolar e sua especialização: uma nova ou antiga atividade. Rev bras enferm [serial on the internet]. 2013 Aug [cited 2019 Nov 01];66(4):607-10. Available from: https://www.scielo.br/pdf/reben/v66n4/ v66n4a22.pdf

24. Abreu LDP, Torres RAM, Silva MRF, Araújo AF. Web rádio como ferramenta de diálogo em saúde coletiva no sertão: juventudes e métodos contraceptivos. Sanare (Sobral, Online) [serial on the internet] 2018 [cited 2021 Jun 11];17(1):2431. Available from: https://sanare.emnuvens.com. $\mathrm{br} / \mathrm{sanare} / \mathrm{article} / \mathrm{view} / 1219 / 650$

25. Silva MAI, Mello FCM, Mello DF, Ferriani MGC, Sampaio JMC, Oliveira WA. Vulnerabilidade na saúde do adolescente: questões contemporâneas. Ciênc Saúde Colet [serial on the internet]. 2014 Feb [cited 2019 Nov 01];19(2):619-27. Available from: http://www.scielo.br/scielo.php?script=sc i_arttext\&pid $=S 141381232014000200619 \& \operatorname{lng}=$ en

26. Baggio MA, Berres R, Gregolin BPS, Aikes $S$. Introduction of the School Health Program in the city of Cascavel, Paraná State: report of nurses. Rev Bras Enferm [serial on the internet]. 2018 [cited 2019 Nov 06];71(Suppl 4):1540-47. Available from: $\quad$ http://www.scielo.br/scielo.php?scrip $\mathrm{t}=\mathrm{s} \mathrm{c}$ i a r t t e $\times \mathrm{t} \& \mathrm{p} \mathrm{i} d=\mathrm{s} 00034$ $\underline{71672018001001540 \& \operatorname{lng}=p t}$
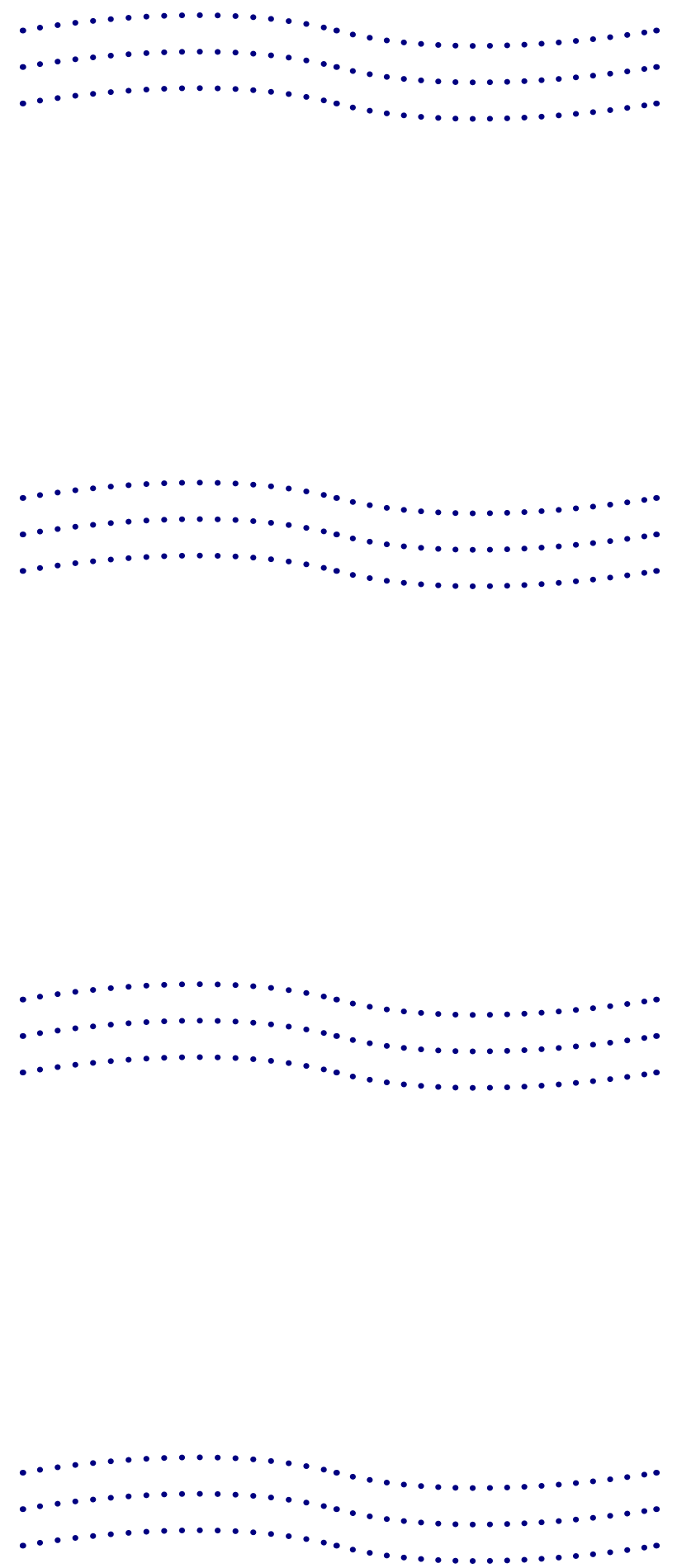